\title{
Criminologie
}

\section{Le génocide à l'épreuve des massacres de masse contemporains : vers une rupture paradigmatique ?}

\section{Samuel Tanner}

Volume 39, numéro 2, automne 2006

Le crime de génocide : Construction d'un paradigme pour la criminologie, la philosophie et le droit pénal

URI : https://id.erudit.org/iderudit/014427ar

DOI : https://doi.org/10.7202/014427ar

Aller au sommaire du numéro

\section{Éditeur(s)}

Les Presses de l'Université de Montréal

ISSN

0316-0041 (imprimé)

1492-1367 (numérique)

Découvrir la revue

Citer cet article

Tanner, S. (2006). Le génocide à l'épreuve des massacres de masse contemporains : vers une rupture paradigmatique ? Criminologie, 39(2), 39-58. https://doi.org/10.7202/014427ar
Résumé de l'article

La violence de masse, considérée ici comme un ensemble d'agressions collectives et étendues, menées contre une population civile, constitue un enjeu majeur de sécurité au XXI ${ }^{\mathrm{e}}$ siècle. Dès lors, l'élaboration d'outils analytiques nous permettant d'en saisir la nature s'avère cruciale. Dans le contexte de cet article, nous proposons l'idée selon laquelle le génocide en tant que paradigme, jusqu'alors utilisé pour saisir ce phénomène, ne présente qu'une valeur heuristique relative à l'épreuve des faits contemporains de massacres tels qu'ils se sont produits en Bosnie-Herzégovine (BiH) entre 1992 et 1995. Après avoir établi une genèse épistémologique du paradigme de génocide, nous dresserons une brève ontologie de la violence de masse telle qu'elle s'est manifestée en Bosnie. Celle-ci révèle des pratiques et dynamiques d'extermination qui restent en grande partie inexpliquées par la proposition d'un projet politique, étatique et intentionnel d'éliminer un groupe en fonction de son caractère religieux, ethnique, racial ou national. De même que la mise en place d'une structure bureaucratique et d'une chaîne de commandement pour l'exécution de telles politiques, telles que proposées dans le génocide, ne permettent pas de saisir la nature de cette élimination. En conclusion, nous esquissons une série de propositions pour la formulation d'un nouveau paradigme capable de saisir ces dynamiques alors mises à jour.
Ce document est protégé par la loi sur le droit d'auteur. L’utilisation des services d’Érudit (y compris la reproduction) est assujettie à sa politique d'utilisation que vous pouvez consulter en ligne.

https://apropos.erudit.org/fr/usagers/politique-dutilisation/ 


\title{
Le génocide à l'épreuve des massacres de masse contemporains: vers une rupture paradigmatique?
}

\author{
Samuel Tanner \\ Doctorant à l'École de criminologie, \\ Assistant de recherche au Centre international de criminologie comparée (CICC). \\ samuel.tanner@umontreal.ca
}

RÉSUMÉ - La violence de masse, considérée ici comme un ensemble d'agressions collectives et étendues, menées contre une population civile, constitue un enjeu majeur de sécurité au XXI $I^{\mathrm{e}}$ siècle. Dès lors, l'élaboration d'outils analytiques nous permettant d'en saisir la nature s'avère cruciale. Dans le contexte de cet article, nous proposons l'idée selon laquelle le génocide en tant que paradigme, jusqualors utilisé pour saisir ce phénomène, ne présente qu'une valeur heuristique relative à l'épreuve des faits contemporains de massacres tels qu'ils se sont produits en Bosnie-Herzégovine $(\mathrm{BiH})$ entre 1992 et 1995. Après avoir établi une genèse épistémologique du paradigme de génocide, nous dresserons une brève ontologie de la violence de masse telle qu'elle s'est manifestée en Bosnie. Celle-ci révèle des pratiques et dynamiques d'extermination qui restent en grande partie inexpliquées par la proposition d'un projet politique, étatique et intentionnel d'éliminer un groupe en fonction de son caractère religieux, ethnique, racial ou national. De même que la mise en place d'une structure bureaucratique et d'une chaîne de commandement pour l'exécution de telles politiques, telles que proposées dans le génocide, ne permettent pas de saisir la nature de cette élimination. En conclusion, nous esquissons une série de propositions pour la formulation d'un nouveau paradigme capable de saisir ces dynamiques alors mises à jour.

ABSTRACT - Mass violence, considered as a set of widespread and collective aggressions lead against a civil population represents a major security issue of the $21^{\text {st }}$ Century. As such, the elaboration of analytical tools allowing us to understand its nature remains crucial. This article proposes the idea that genocide, as a prevalent paradigm used to grasp that phenomenon, holds relative heuristic value confronted to contemporary massacres such as the events that took place in Bosnia-Herzegovina between 1992 and 1995. Firstly, we draw a genesis of the genocide and then some of the epistemological sources that established it as a paradigm. Then, a short ontology of mass violence that 
happened in Bosnia will be sketched out. This will lead us to the observation that some extermination patterns remain unexplained by the intent to destroy in whole or in part a group based upon their ethnical, national, religious or racial membership; or by a bureaucracy and a chain of command such as proposed by the genocide paradigm. To conclude, we draw a few propositions to help the elaboration of a new paradigm able to grasp the new patterns revealed.

\section{Le génocide comme paradigme}

Lorsqu'un ensemble de propositions émerge formant un consensus à partir duquel se développe une tradition de recherche scientifique autour d'un objet, Thomas S. Kuhn (1962) parle de paradigme. Aussi, et à l'intérieur d'une discipline, des théories et modèles sont peu à peu élaborés par des pratiques scientifiques (science fondamentale, science appliquée) qui se systématisent pour fonder des traditions de recherche. Un paradigme ou langage dans lequel sont formulées les théories ou éventuellement des sous-ensembles importants de théories émises dans le cadre d'une discipline, se forme (Boudon, 1977: 190). Un tel cadre suppose un ensemble de règles et de conventions où ce qui doit être observé, le type et le mode de validation des questions posées quant à l'objet étudié et enfin, le mode d'interprétation des résultats, sont préétablis.

En matière d'étude de massacres de masse, le génocide constitue un paradigme important du $\mathrm{XX}^{\mathrm{e}}$ siècle. Certains l'utilisent rétrospectivement pour apporter une lecture nouvelle de cas historiques, tels que le massacre des habitants de Mélos par les Grecs au Ve siècle avant J.-C. ou des Amérindiens (Chalk et Jonassohn, 1990). Ce paradigme voit sa formulation la plus aboutie dans la Convention pour la prévention et la répression du crime de génocide des nations unies, ratifiée en 1948, où le génocide est envisagé comme une intention de détruire, en tout ou en partie, un groupe eu égard à sa race, religion, ethnicité, nationalité. Or, pour la discipline traitant de l'élimination d'une population d'un territoire, cela revient à supposer que le pouvoir législatif demeure l'autorité exclusive capable «de dire officiellement comment nommer et penser un événement». Cette situation est intolérable selon Jacques Sémelin pour qui la loi ne peut prétendre à «qualifier l'Histoire [et édicter] la vérité historique» (Sémelin, 2005: 368). Nous souhaitons joindre notre voix à celle de ce chercheur en ajoutant que la loi, pas plus qu'une perspective réaliste envisageant une lecture de tels phénomènes exclusivement sur le plan étatique, ne peuvent édicter la vérité 
épistémologique en matière d'appréhension des massacres de masse. Leur étude ne doit pas être fondée exclusivement sur un texte juridique, et donc normatif. Elle doit au contraire faire appel à une pluridisciplinarité (sociologie, sciences politiques, anthropologie, criminologie) qui permettra de problématiser davantage le champ des études sur le génocide. L'élimination d'une population ne peut être conçue en termes exclusifs de chaîne de commandement et d'un continuum entre des leaders synchronisant des exécutants, mais bien davantage comme un processus engendrant ses propres dynamiques et produisant en grande partie sa propre morphologie.

La notion de génocide a cette particularité de s'entendre de deux façons. À la fois crime au regard du droit international, elle renvoie aussi à des pratiques sociales de violence de masse contre une population. Nous distinguerons alors le génocide des massacres de masse, l'un faisant exclusivement référence au crime et à la violation d'une règle de droit, les autres désignant les pratiques sociales de violence de masse ayant leur propre ontologie et formant ainsi un objet sociologique. Aussi, quelle valeur heuristique le paradigme de génocide détient-il à l'épreuve des massacres de masse contemporains et, le cas échéant, quels outils conceptuels de recherche doivent être conçus pour en accroître leur compréhension? Quant à l'élimination, celle-ci peut à la fois être envisagée comme la destruction physique d'un groupe, tel qu'exclusivement impliqué dans la définition du génocide, mais également comme une déportation ou tout autre moyen visant à faire disparaître, symboliquement (destruction de la culture) ou physiquement (déportation), une population d'un territoire. Les pratiques de violence de masse débordent alors de la stricte extermination d'un groupe. En conséquence, l'idée d'une grille de lecture basée exclusivement sur le paradigme de génocide comme nous le définirons ci-dessous, à la fois centré sur l'extermination et sur une perspective réaliste dont l'État représente l'unité analytique, ne permet plus à elle seule de saisir l'ontologie de ces violences de masse. Comprendre plus justement ce phénomène dans l'espoir de prévenir, sinon de contribuer à sa désescalade, requiert la réalisation de nouveaux outils heuristiques.

Dans un premier temps, nous définirons la notion de génocide et mettrons à jour l'ensemble des propositions qui ont structuré le champ d'étude de l'élimination d'une population. Nous présenterons alors les sources épistémologiques majeures qui ont donné au paradigme de génocide sa substance et lui ont conféré son statut en tant qu'outil 
analytique exclusif de l'étude du massacre de masse au $\mathrm{XX}^{\mathrm{e}}$ siècle. Une seconde section contribuera à l'édification d'une ontologie de la violence de masse, de sa substance, par la présentation de recherches ayant mis au jour des dynamiques locales ayant entrainé l'homogénéisation ethnique dans certaines régions de Bosnie-Herzégovine. Enfin, nous proposerons une réflexion pour la création d'un nouveau modèle de recherche en sciences sociales pour l'étude de ce phénomène dont la valeur heuristique sera, nous l'espérons, accrue. Nous pouvons déjà préciser que le propos consiste moins à remettre en question la nature juridique qu'heuristique du génocide tel qu'envisagé jusqu'à maintenant.

\section{Le génocide et ses sources épistémologiques}

\section{Un concepteur: Raphaël Lemkin}

Le génocide, terme créé par Raphaël Lemkin (1901-1959), alors réfugié aux États-Unis lors de la Seconde Guerre mondiale, provient de la juxtaposition du grec genos (race) et de la particule latine -cide (tuer). Il désigne «un plan coordonné de différentes actions dont le but vise la destruction des fondations vitales de groupes nationaux» (Lemkin, 1944: 82). Souhaitant mettre en équation de nouvelles conceptions avec des pratiques modernes d'élimination d'une population et, qui plus est, témoin des atrocités menées contre la population civile arménienne et des pratiques nazies en cours, Lemkin comprend rapidement que les concepts de dénationalisation, germanisation ou italianisation désignant alors ces agressions ne suffisent pas à en saisir la nature. Pour le juriste, il n'est pas tenu compte des dimensions biologique et physique ${ }^{1}$, qui pourtant constituent une dimension majeure des attaques. Ces pratiques, conclut l'auteur, suivent un pattern récurent consistant en une atteinte globale et systématique aux dimensions politique, sociale, économique, physique, biologique, religieuse, culturelle et morale, qu'il regroupe sous la notion de génocide.

1. Sur le plan biologique, Lemkin fait référence aux moyens employés pour ralentir et faire chuter les taux de natalité des groupes à éliminer et édicter des politiques permettant au groupe privilégié d'accroître les naissances. D'autres mesures consistaient à maintenir les populations en état constant de sous-nutrition et d'empêcher les mariages mixtes. Quant à la dimension physique, elle consistait essentiellement en une mise en danger systématique de la santé des groupes à éliminer ainsi qu'en des pratiques de massacre de masse. 
Cependant, saisir le caractère même des actions menées contre la population ne constituait que la première étape d'une démarche qui se révéla avant tout juridique. Il s'agissait pour Lemkin de fournir un ensemble de propositions, une description la plus complète qui soit, pour saisir le mal absolu et l'horreur la plus extrême que l'homme soit capable d'infliger à ses semblables, bref, de fournir la substance suffisante à la création d'un crime de droit international. Ses efforts ont en partie abouti à la ratification de la Convention des Nations Unies, en 1948. Bien qu'outil majeur de prévention de ce type de violences, les idées du juriste ne s'y retrouvent que partiellement et seules les dimensions physique et biologique de la destruction ont été retenues ${ }^{2}$. Mais concentrons-nous davantage sur les sources épistémologiques, ou fondements, qui ont donné au paradigme de génocide sa substance. Nous laisserons le soin au lecteur intéressé par les préoccupations juridiques de Lemkin de consulter les nombreux ouvrages traitant de cette question (Kuper, 1981; Ball, 1999; Schabas, 2000).

\section{Le réalisme en relations internationales et la question de l'État}

Le principe selon lequel, seuls l'État et ses institutions disposent de la force et de la puissance nécessaires pour commettre de tels massacres n'est peu, voire pas du tout remis en question. Il représente une constante dans la documentation traitant de ce sujet (Arendt, 1963; Kuper, 1981; Hilberg, 1985; Horowitz, 2002). Cela tient en grande partie d'un courant de pensée exerçant une influence décisive sur l'étude de la violence de masse: le réalisme en relations internationales. Il se fonde sur la transposition, à une échelle internationale, de l'idée de Thomas Hobbes (1651) selon laquelle l'État constitue l'unité politique de base en deçà et au-delà de laquelle l'analyse politique est impossible. Hobbes entrevoit l'État (le Léviathan) comme le seul moyen de faire cesser la «guerre de tous contre tous», d'assurer l'accomplissement d'un projet

2. Cette convention définit le génocide comme: «l'un quelconque des actes ci-après commis dans l'intention de détruire en tout ou en partie un groupe national, ethnique, racial ou religieux: a) meurtres des membres du groupe; b) atteintes graves à l'intégrité physique ou mentale de membre du groupe; c) soumission devant entraîner la destruction physique totale ou partielle [du groupe]; d) entrave des naissances; e) transfert forcé d'enfant du groupe à un autre groupe». 
commun dont la valeur dépasse les intérêts de chacun, et d'ainsi pallier l'état de nature et de chaos qui caractérisent les relations humaines présupposées en son absence. À cette idée se joint une vision civilisatrice et de progrès humain. Mais l'État est perçu comme fondamentalement agressif, en quête de nouveaux espaces et d'expansion territoriale argument que les analystes réalistes ont alors mis de l'avant pour expliquer la politique du Lebensraum appliquée par le III ${ }^{e}$ Reich (Hilberg, 1985), ainsi que la politique d'épuration ethnique par les dirigeants serbes (Grmek et al., 1993; Silber et Little, 1995; Denitch, 1996).

Max Weber, pour qui l'État se définit par un monopole de l'usage de la force légitime sur les citoyens ainsi que par une bureaucratie rationnelle et minutieuse, constitue une référence incontournable de ce courant (Weber, 1921). La politique est un acte de pouvoir, lequel se distribue d'une manière toujours favorable à l'État. Mais pour maintenir son hégémonie, celui-ci est obligé de décharger une partie de ses prérogatives à un ensemble d'agents désignés de la collectivité (police, armée, services de renseignements). Ceux-ci bénéficient alors d'un statut réglementé, légitime et constitutionnellement fondé qui les distingue du citoyen ordinaire, notamment par le droit à l'usage de la force légitime sur quiconque viole l'ordre établi. Aussi, l'impulsion guidant l'ensemble de la société provient systématiquement d'une domination du haut de l'organisation sur le bas, dernier échelon d'une chaîne de commandement formelle et rationnelle. Fort de telles prémisses, le génocide est considéré comme une pratique propre à l'État et ses dirigeants qui, à travers une chaine de commandement complexe, commettent une agression sur une partie désignée de ses citoyens (Horowitz, 2002; Kressel, 2002). Même si un certain jeu (Sémelin, 2005) peut exister entre les gouvernants et les exécutants, qui disposent d'un degré d'autonomie dans le mode d'exécution, les ordres sont systématiquement issus des dirigeants. À cet égard, C. Browning (1993) a montré dans une étude remarquable que des acteurs en périphérie constitués des policiers de réserve du bataillon 101, notamment les Einzatzgruppen, tenaient un rôle décisif dans l'extermination de populations lors de l'avancée de la Wehrmacht vers l'est, en 1941. En effet, et constatant le «faible rendement» des fusillades en pelotons d'exécution, les policiers prirent l'initiative de faire usage de nouvelles techniques d'assassinats, dont notamment les chambres à gaz mobiles, permettant un rendement accru du processus d'extermination. Si l'État tient bien entendu une responsabilité immédiate dans de telles pratiques, nous verrons par la 
suite que, dans le contexte de la $\mathrm{BiH}$, une part de l'agression reste inexpliquée par une perspective de type wébérien.

Carl Schmitt (1993) prétend que si l'État demeure l'unité de toute réflexion sur l'organisation sociale ou les relations internationales, celuici ne peut se penser sans tenir compte de la notion de politique, laquelle constitue le champ même d'intérêt le définissant. En d'autres termes, s'il est un domaine dans lequel l'État agit, c'est précisément la politique et les deux notions ne peuvent se concevoir sans faire référence à l'autre. Schmitt fonde la politique sur un principe de discrimination ami/ ennemi qui a valeur de critère d'identification pour les populations. Le statut d'ennemi est attribué sur une base existentielle, autrement dit relativement à la réalité vécue par chacun. Ainsi l'autre peut symboliser une menace telle aux yeux du groupe qu'il devient nécessaire de le combattre et l'éliminer. Dès lors, pour Schmitt, la violence s'explique par l'appartenance à des loyautés et des croyances (univers symbolique) qui déterminent cet autre avant même qu'il ne soit abordé, rencontré sur une base concrète. Par la mise en place de législations ou mesures administratives, l'État contribue à la formation et la cristallisation de ces loyautés, allégeances et croyances, entérinant ainsi la polarisation des groupes qui peut engendrer la violence extrême selon Schmitt. Qu'elle soit idéologique ou révolutionnaire, la politique confère un sens à la violence qui peut ainsi s'interpréter, se justifier et se perpétrer. Holocauste, nettoyage ethnique, génocide au Rwanda sont tous des épisodes où la violence se voit légitimée aux yeux de ses exécutants par des différences perçues et élevées au rang d'inimités irréversibles, réelles menaces qui, selon le principe «eux ou nous», nécessitent alors l'élimination de l'autre (Goldhagen, 1997; Judah, 1997; Hatzfeld, 2003). Par exemple, la tragédie en $\mathrm{BiH}$ a, en grande partie, été lue en termes de haines ethniques ou nationalistes irréversibles (Malcolm, 1994 ; Denitch, 1996; Hayden, 2002). Cette lecture n'est pas suffisante dans la mesure où non seulement certaines milices mélangeaient plusieurs clans, mais aussi et comme nous le verrons, où une partie des massacres s'explique par des motifs qui n'ont rien d'une polarisation. Enfin, Schmitt mentionne le rôle majeur des dirigeants, puisqu'ils déterminent en grande partie le contenu de ces représentations. On peut penser notamment au rôle du pouvoir dans les médias alors instrumentalisés lors des premiers temps de la guerre en Croatie (Judah, 1997). 


\section{Un cas historique: l'Holocauste}

L'Holocauste constitue le modèle à partir duquel l'ensemble des massacres de masse de la seconde partie du $\mathrm{XX}^{e}$ siècle a été analysé, que ce soit le Cambodge, le Timor-Oriental, le Rwanda ou l'ex-Yougoslavie. Si la naissance d'un paradigme provient en grande partie de la conjonction d'un courant de pensée et d'un événement particulier (Kuhn, 1962), on peut raisonnablement affirmer que le génocide en tant que paradigme est né de la conjonction du réalisme et de l'Holocauste.

Dans le cas de l'extermination menée durant la Seconde Guerre mondiale, les chercheurs mettent au jour une machine sociale et une bureaucratie extrêmement complexe conçue par le régime nazi. L'extermination de millions de Juifs, Tziganes, homosexuels et invalides représente le résultat d'une action sociale rationnellement ordonnée (Lemkin, 1944; Arendt, 1963; Hilberg, 1985). L'identification, l'expropriation, le rassemblement-concentration et, enfin, l'extermination, sont toutes des pratiques réglementées par une législation précise imaginée par le régime de Hitler. Cette machine sociale comportait quatre hiérarchies distinctes: la bureaucratie ministérielle, les forces armées, l'économie et enfin, le parti. Hilberg (1985), ainsi que Arendt (1963), ajoutent que la coopération entre ces différentes autorités fonctionnait parfaitement, entraînant de surcroît une diffusion de la responsabilité. Aussi, Arendt (1963) parle de massacre administratif, en grande partie exécuté par des criminels de bureau et dont la nature massive, par son ampleur, sa répétitivité et sa continuité, ont inspiré sa désormais célèbre thèse de la banalité du mal. C'est justement ce processus administratif qui distingue l'Holocauste d'un pogrom (terminologie alors utilisée à l'époque) dont la nature est plus expéditive. Un lien puissant est alors établi entre les massacres de masse et le rôle de l'État. Et Hilberg de conclure: «Bref, parler de la machine de destruction, c'est parler de l'État allemand considéré dans une de ses fonctions spécialisées» (Hilberg, 1985: 53). «Le génocide n'est pas un acte sporadique ou un événement aléatoire tel que les massacres de la forêt Katyn (durant la Seconde Guerre mondiale) [...] En plus de son caractère systématique, le génocide doit être mené avec l'approbation, si ce n'est pas l'intervention directe de l'appareil étatique» (Horowitz, 2002: 14). Dès lors et conformément aux prédictions de Kuhn: "La mise à mort bureaucratique n'est intelligible que dans le cadre de l'analyse des appareils de pouvoir [étatiques] et des processus de décision» (Brauman et Sivan, 1999: 18). Si seul 
l'État est capable de mettre à exécution un tel programme, les responsabilités que les procès de Nuremberg établiront toucheront essentiellement ses serviteurs (dirigeants et fonctionnaires) et leur responsabilité dans la chaîne de commandement.

\section{Un processus: la modernité}

La modernité constitue un élément central dans l'étude des massacres de masse. Elle demeure cependant un concept complexe dont nous ne retiendrons que quelques dimensions essentielles à notre propos. Si elle coïncide avec l'émergence de l'État-nation au milieu du $\mathrm{XV}^{\mathrm{e}}$ siècle, c'est avant tout par la laïcisation et l'apparition de la pensée rationnelle, qui marque une rupture avec les activités intellectuelles jusqu'ici essentiellement influencées par la superstition et la religion, que la modernité constitue une étape fondamentale dans l'évolution de la pensée humaine. Cette rupture voit émerger de nouvelles connaissances qui entraînent un essor rapide des sciences, de la technologie et contribuent intensément au progrès de l'humanité. Mais c'est avant tout en matière d'organisation sociale que la modernité nous intéresse. Un processus de différenciation sociale fait émerger des configurations gouvernementales qui se hiérarchisent, se systématisent et font apparaitre une bureaucratisation alambiquée ainsi qu'une division du travail qui se complexifient. L'idée de l'ingénierie sociale fait son apparition où Max Weber parlera de substitution du concept de société (Gesellschaft) à celui de communauté (Gemeinschaft). Les relations humaines ne s'organisent non plus en vertu d'une tradition et de données de nature religieuse ou sacrée, mais bien plutôt par un calcul rationnel de leurs intérêts communs. Ils sont désormais mus par une finalité et l'espoir d'obtenir des règlements qui leurs soient les plus profitables (Pisier et al., 2004). La bureaucratie annonce le passage de l'action communautaire à l'action sociale, rationnellement ordonnée, et constitue dorénavant l'instrument de socialisation des relations de pouvoir. Cette organisation ne tolère que peu l'initiative privée ou périphérique, notamment en matière d'élimination d'une population (Arendt, 1963).

La modernité envisage une étiologie des massacres de masse fondée sur un projet rationnel bien plus qu'émotionnel ou sacré. Une sémantique moderne a remplacé un champ lexical barbare où les massacres sont analysés par les notions de technique, rationalité, bureaucratie, 
distanciation morale, division du travail, tâches techniques désormais privilégiées ${ }^{3}$. Par exemple, Zygmunt Bauman (1989: 26) prétend que: «Le succès technico-administratif de l'Holocauste a été en grande partie dû à l'utilisation judicieuse de "somnifères moraux» rendus disponibles par la bureaucratie moderne et la technologie moderne». Et d'ajouter: «La lumière jetée par l'Holocauste sur nos connaissances de la rationalité bureaucratique est à son paroxysme une fois que l'on réalise l'étendue à laquelle l'idée de "Solution finale" n'était qu'un produit de la culture bureaucratique» (Bauman, 1989: 15). Les explications modernes auront su faire un lien essentiel entre une perspective schmittienne de la violence, dont l'étiologie remonte à un conflit majeur entre deux groupes antagonistes, et le dispositif par lequel elle se répand, État, bureaucratie, chaîne de commandement. Aussi, l'Holocauste et les massacres de masse du $\mathrm{XX}^{\mathrm{e}}$ siècle sont envisagés comme la conséquence d'une absorption sans précédent d'un pouvoir de coercition par l'État et sa bureaucratie, qui disposent de moyens rationnels extraordinaires pour mener à bien un projet criminel. Mais une autre face de la modernité est révélée, qui vient pour le moins jurer avec la naïveté du métarécit civilisateur qui l'accompagnait (Bauman, 1989; Traverso, 2004). Si Thomas Hobbes voyait en la création du Léviathan un moyen de faire cesser la violence de tous contre tous et d'assurer protection et prospérité à ses citoyens, les événements du $\mathrm{XX}^{\mathrm{e}}$ siècle représentent une rupture ou une pondération de la tradition civilisatrice alors associée à la modernité.

La compréhension des massacres de masse de populations civiles est alors formulée en termes de chaîne de commandement par le haut, de bureaucratie étatique, d'ingénierie sociale et de projet rationnel soustendu par une idéologie de race, d'ethnicité, de religion ou de nationalité. Ces axes ont formé un paradigme qui a outillé de nombreux auteurs dans la période suivant l'Holocauste (Kuper, 1981; Hilberg, 1985; Walliman et Dubkowski, 1987; Chalk et Jonassohn, 1990; Fein, 1990; Andreopoulos, 1994; Malcolm, 1994; Ball, 1999; Robertson, 1999; Horowitz, 2002; Kressel, 2002). Cependant, comme nous allons le voir dans la section suivante, les contextes contemporains dressent un

3. Du reste, ce champ lexical a parfois desservi la compréhension du phénomène dans la mesure où les catégories d'évaluation de l'élimination d'une population n'ont pour certains plus permis de faire une distinction entre un génocide et un crime de guerre, tel que les bombes lâchées sur Hiroshima et Nagasaki (Charny, 1988). 
répertoire différent des pratiques d'élimination d'une population. Les violences qui se sont produites en Bosnie-Herzégovine entre 1992 et 1995 restent en grande partie inexpliquées par le paradigme du génocide et de nouveaux outils analytiques sont requis. Une question fondamentale se pose pour le champ des études sur le génocide qui touche à l'objectif qu'elles se fixent: doit-on poursuivre l'étude des massacres de masse en restant préoccupé par une perspective par le bas, la position de l'État, la politique ou la bureaucratie dans l'élimination d'une population? Ou plutôt, n'est-il pas plus pertinent d'initier la réflexion en se posant la question des dynamiques, logiques, processus par lesquels la population civile est massacrée, supposant alors un renversement conceptuel et l'apparition d'une perspective par le haut, de l'interaction des dynamiques locales avec les logiques étatiques (Kalyvas, 2003)? Autrement dit, n'est-il pas plus adapté de s'intéresser non plus à une perspective d'élimination d'une population comme une continuité entre des dirigeants et des exécutants, mais bien à la part d'autonomie dont ceux-ci jouissent et aux dynamiques qui caractérisent la participation à une activité de violence de masse? Plutôt que de considérer l'État et ses institutions comme unités analytiques principales, il est désormais nécessaire de prendre pour point de mire la violence même et ses dynamiques. Procédons à l'exposé d'une série de logiques locales qui nous permettront de réfléchir sur de nouvelles propositions.

\section{Un répertoire contemporain différent: le cas de la Bosnie}

Dans le contexte des événements qui se sont produits en BosnieHerzégovine entre 1992 et 1995, nous allons montrer qu'une partie des massacres suit des dynamiques différentes de l'Holocauste. Nous concentrerons notre analyse sur deux axes: les acteurs-auteurs de la violence et l'univers des motivations qui semble les avoir animés.

\section{Une pluralisation d'exécutants}

De nombreuses recherches ont montré que si l'armée populaire yougoslave, alors passée aux mains des dirigeants serbes avec Slobodan Milosevic à leur tête, était indiscutablement impliquée dans les massacres de masse, le pouvoir et l'usage de la violence se répartissaient selon 
une distribution complexe impliquant une nébuleuse de gens armés (Bassiouni, 1994; Bougarel, 1996; Loyd, 1999; Mueller, 2000; Ron, 2000b; Bax, 2000a; Stroschein, 2005). En premier lieu, on dénombre une série d'organisations paramilitaires très bien structurées, engagées par les services de sécurité des différents régimes nationalistes d'alors (Ron, 2000a). Parmi ces formations, relevons les Tigres de Zeljko Raznjatovic, dit Arkanl; les Bérets rouges, unité spéciale de la police serbe; ou encore le Conseil de défense croate (HVO) branche armée des Croates de Bosnie-Herzégovine. Mais à cela s'ajoute un nombre important de groupes armés plus difficilement reconnaissables, comme le relève A. Loyd, alors journaliste couvrant la guerre à Sarajevo:

La ville était pleine de pièges, les structures de pouvoir ainsi que les allégeances étaient loin d'être évidentes, même pour ceux qui y habitaient. Le combat a, dans un premier temps, brisé puis effacé la hiérarchie de l'autorité et de la structure sociale [...] En l'absence d'une armée professionnelle, les seuls groupes disposant d'un minimum d'organisation, d'armement et de structure, n'étaient autres que les gangs de criminels de la ville $[\ldots]$ (Loyd, 1999: 24) ${ }^{4}$.

Les Nations Unies relèvent la présence de 83 groupes armés différents ayant été actifs en Bosnie-Herzégovine dans les deux premières années du conflit, et dont il est précisé qu'ils ont endossé une responsabilité majeure dans les massacres (Bassiouni, 1994; Mueller, 2000). Xavier Bougarel (1996) ajoute que, parmi ces groupes, une grande proportion agissait en dehors de toute chaîne de commandement et présentaient des caractéristiques pour le moins éloignées de la figure du soldat ou de celle du guérillero. À ce titre, John Mueller (2000) parle de groupes de voyous, de brutes, supporters de football, d'opportunistes, maraudeurs n'étant mus par aucune idéologie si ce n'est l'appât du gain. S'ajoutent à cela des détenus coupables d'homicides pour lesquels les portes ont été ouvertes et des rémissions de peines ou libérations, promises contre leur participation aux violences. Une partie de ces individus a été recrutée par des partis nationalistes, à travers ce que James Ron (2000a) nomme la ligne militaire (military line). Processus parallèle aux structures de l'État, elle visait à pallier le problème de la désertion massive des soldats refusant de participer au projet criminel, mais aussi à démentir toute implication officielle de l'État dans les

4. Les traductions sont de l'auteur. 
massacres. L'initiative privée de ces bandes armées en matière d'assassinat, de pillage, de viol, autant de stratégies provoquant la terreur et l'exode des populations, n'entraînait aucune sanction de la part des dirigeants qui les cautionnaient sans qu'ils ne les guident pour autant sur le terrain. Le répertoire de la violence telle qu'elle s'est produite en Bosnie différait également sur une autre dimension qui demeure inexpliquée par le paradigme de génocide. Par exemple, comme le mentionnent Loyd (1999) ainsi que Bassiouni (1994), une grande partie de ces groupes agissait en dehors de tout commandement étatique ou politique:

Durant longtemps, la stratégie gouvernementale reposait dans les mains de criminels durs et énigmatiques bénéficiant d'un culte local et d'un goût pour les tueries. Plus tard cette année [1993] les batailles n'étaient pas menées entre les tranchées avec les Serbes, mais à l'intérieur même de Sarajevo lorsque le gouvernement chercha à reprendre le contrôle des mains de ces groupes mafieux fragmentés [...] (Loyd, 1999: 24).

Durant les premières phases des conflits en Croatie et en Bosnie-Herzégovine, la police, aidée de civils armés volontaires, a opéré sans commandement ni contrôle apparent de l'armée. Son leadership était local et incluait de nombreuses figures politiques. Ces différents groupes ont alors agi dans une autonomie quasi complète (Bassiouni, 1994: 39).

Rappelons cependant que le contexte étatique de la Bosnie-Herzégovine de l'époque reste difficilement comparable à celui de l'Allemagne nazie. Si dans le cas de cette dernière les institutions étatiques fonctionnaient parfaitement, tel n'était pas le cas en Bosnie où la plupart des structures institutionnelles se sont effondrées dès le début de la guerre. Et si le président de la Bosnie, Alija Izetbegovic, a tenté de maintenir un étatmajor fonctionnel pour organiser une défense contre l'agression serbe et croate, très rapidement l'armée et ses rangs se sont "factionnalisés», perdant ainsi tout contrôle sur une grande partie des hommes armés.

Il n'en demeure pas moins que les violences se sont poursuivies, et ces données suggèrent une reconfiguration majeure du pouvoir et de l'usage de la force. Les massacres, dans le contexte de la Bosnie, impliquent un répertoire d'exécutants outrepassant la stricte participation de l'État et sa bureaucratie. Dès lors, l'élimination de populations civiles se manifeste par un champ de pratiques multilatérales qui mobilise une pluralisation d'exécutants de nature différente: armée, milices, gangs criminels, groupes paramilitaires, clans locaux, collaborateurs. Cette action conjointe entraine une complexification des dynamiques de la 
violence, comme nous allons le voir dans la section suivante, et en multiplie les foyers. Ces données suggèrent alors, sans l'infirmer pour autant, que le paradigme de génocide ne permet pas de saisir les dynamiques et processus qui caractérisent l'élimination de populations comme le résultat exclusif de la mise en application d'un plan décidé par les dirigeants. Ces violences suivent une dynamique de réticulation où le processus décisionnel menant aux violences se pluralise.

\section{Une pluralisation des dynamiques locales de la violence}

Des travaux adoptant une perspective anthropologique et locale ont révélé que des massacres s'étant produits dans certaines régions de Bosnie sont davantage liés à une influence locale que le résultat d'une coordination politique. Mart Bax (2000a) relève le rôle crucial du clergé catholique franciscain dans la région de Mostar qui, à la suite de l'effondrement des structures étatiques de Bosnie-Herzégovine et du monopole de l'usage de la force par un État central, sont, en plus de leur statut de chefs religieux, devenus des leaders séculiers locaux. Rejetant la thèse d'une stricte planification de la violence "par le haut», l'auteur prétend que, sans pour autant parler de balkanisme primitif (Malcolm, 1994; Silber et Little, 1995), ces violences constituent le résultat de pratiques de vendettas entre communautés locales, qui pourraient bien avoir été planifiées, mais n'étaient pas ethniquement fondées. Ces vendettas, ajoute-t-il, étaient conduites sur les mêmes axes que les conflits séculaires entre communautés religieuses, abondamment décrits dans l'article. Il ne s'agit pas d'éclats spontanés et cette violence est avant tout le résultat de dynamiques rationnelles. Bax montre que le rôle et l'autorité des frères franciscains s'expliquent en grande partie par la volonté de la population paysanne et bergère, systématiquement sous le joug des Serbes et des Musulmans, de prendre la situation en main et se défendre. En tant que leaders locaux, les frères franciscains, grâce à leurs réseaux, ont alors organisé des formations militaires ainsi que la fourniture d'armement. Ces moines ont aussi fait office de guides pour des formations militaires dont le Conseil de défense croate (HOS), ainsi qu'une milice locale, les Roquettes, dirigée par un entrepreneur politico-mafieux local alors en charge de la protection des frères. Dès lors, et plus que les institutions étatiques, c'est le clergé qui endossa un rôle stratégique dans l'élimination de population et l'homogénéisation de plusieurs régions du sud-ouest de la Bosnie-Herzégovine. 
Dans une autre recherche portant sur un lieu de pèlerinage catholique célèbre, à Medjugorje, dans le sud-ouest du pays, Bax (2000b) analyse la manière dont la région a ethniquement été homogénéisée à la suite de la guerre. Encore une fois, l'auteur montre qu'une telle épuration ethnique n'est pas le résultat d'une politique militaire planifiée d'en haut, mais plutôt de vendettas entre factions locales qui s'insèrent dans un contexte d'industrie touristique liée d'une part aux enjeux que représente le pèlerinage et, d'autre part, à la faillite de l'État en matière de monopole de la violence et d'arbitrage de querelles privées. Tout au long de son article, Bax analyse les rivalités entre les deux camps présents dans la région à travers les âges sans pour autant qu'il ne soit question de haines ancestrales. Bax (2000b) évoque alors le concept local de mali rat (petite guerre) pour désigner les opérations plus ou moins violentes que ces clans ou familles se sont livrées, notion basée sur la vengeance du sang, et qui ont abouti au quasi-anéantissement de l'un des clans. Selon Bax:

[Ces résultats] indiquent qu'il s'agit d'une expansion et intensification temporaire de ce qui est essentiellement un processus en cours de maintien et de reproduction d'antagonismes extrêmement passionnés entre et parfois à l'intérieur même de groupes locaux (Bax, 2000b: 311).

Et l'auteur d'ajouter:

[S]elon mes informateurs, la mali rat peut être un effet secondaire de la guerre organisée sur une échelle plus large mais n'est pas équivalente $[. .$. Elle est liée à une étape de la formation de l'État qui est caractéristique de certaines régions de l'ex-Yougoslavie où le monopole sur les moyens de la violence organisée reste ineffectif et non centralisé (Bax, 2000b: 334).

Mais surtout, l'auteur indique que la référence à l'ethnicité doit être pondérée par le fait que celle-ci est flexible et qu'elle ne doit en aucun cas être interprétée comme scellée une fois pour toute. En effet, les deux clans en question étant à l'origine croates, il est crucial de noter que l'un d'eux étant désigné comme serbe par son rival pour avoir demandé de l'aide à la police (serbe dans cette région) a ainsi subi un dénouement tragique dès l'arrivée de troupes militaires croates qui, affirmant aider leurs frères de Medjugorje, ont alors «nettoyé» le village des Serbes désignés. Et Bax de conclure:

En bref, les problèmes de Medjugorje n'étaient pas ethniques à l'origine. Mais plutôt, ils ont été ethnicisés au cours du développement des événements [...] Ainsi, la notion récurrente d'identités ethniques fermement 
établies comme étant la cause même des problèmes récents des Balkans est une simplification et une représentation inadéquate de la réalité, soigneusement propagée par les dirigeants politiques nationaux et adoptée sans réserve par les scientifiques et les journalistes étrangers (Bax, 2000b: 332).

De telles constatations trouvent également un écho dans une recherche menée par James Ron dans la région du Sandjak, à cheval sur les territoires de la Bosnie-Herzégovine et de la République fédérale yougoslave (RFY), Serbie et Monténégro d'alors. L'auteur montre que si les Musulmans vivant sur le territoire de la Bosnie étaient systématiquement massacrés par des paramilitaires ainsi que des bandes armées serbes, tel n'était pas le cas de leurs frères vivant sur le territoire de la RFY, bien que régulièrement harcelés (Ron, 2000b). Est alors remis en cause l'argument selon lequel l'étiologie des massacres correspond à une haine ethnique mais bien plutôt à une guerre d'agression. Enfin, et pour terminer ce panorama non exhaustif, S. Kalyvas $(2003$; 2006) avance l'idée selon laquelle dans certains cas (les guerres civiles, par exemple), une grande part des violences résulte de transactions entre les adversaires (groupes armés, criminels) et les gens ordinaires qui, pour des raisons d'ordre privé bien plus qu'idéologiques, participent conjointement aux massacres par la pratique de la dénonciation. Ainsi donc, les griefs personnels comptent parmi la pluralisation de motifs de la violence de masse. Celle-ci suit un processus rarement hasardeux et reste la plupart du temps soumise à des formes de régulation, quel que soit leur niveau organisationnel: étatique, institutionnel ou individuel. Des configurations sociales se forment, parfois sur base ad hoc, qui posent de nouveaux défis aux chercheurs des études sur le génocide. Dès lors, une étiologie de la violence de masse doit tenir compte du fait que celle-ci n'est pas forcément la conséquence de haines ethniques profondes ou le résultat de la mise en application d'un projet politique rationnel. Elle peut s'alimenter de microcontentieux entre voisins, amis, provoquant ainsi ce que Bougarel (1996) appelle le crime intime.

\section{Conclusion}

Une microanalyse des massacres de masse qui se sont produits en Bosnie fait apparaître des logiques plurielles qui diffèrent du répertoire mis à jour par l'Holocauste. L'attention portée exclusivement sur l'État 
ne suffit pas à saisir une grande proportion des dynamiques locales de la violence qui se situent en aval des institutions étatiques, à la fois au regard du type d'acteurs impliqué et de leurs motivations. En outre, et contrairement au cas d'école que représente la Shoah, le mode opératoire des massacres de masse de Bosnie ne laisse pas présager d'une stricte bureaucratie rationnelle et minutieuse régulant l'ensemble des relations sociales et des rapports de pouvoir entre les acteurs des différents échelons. Enfin, les tactiques et dynamiques exposées ci-dessus, quoique non exhaustives, ne permettent pas de conclure à un massacre dont l'étiologie reposerait simplement sur une dialectique ami/ennemi telle qu'envisagée par Schmitt (1963), ni ne correspondent pour autant à l'expression de l'état de nature hobbesien. Ces violences s'expliquent en grande partie par une pluralisation de microrationalités ou microrégulations qui les gouvernent et dont le mali rat en constitue une expression. Ces massacres constituent une interface entre un redéploiement des sphères politiques, privées, étatiques et non étatiques dont le dénominateur commun semble être un critère «massif» et qui s'interpénètrent selon des configurations nouvelles. Ils sont massifs puisqu'il s'agit avant tout d'actions collectives; qu'ils impliquent une pluralisation d'acteurs agissant à grande échelle, à la fois au regard du nombre de victimes et de leur distribution sur le territoire; se répètent et s'étendent sur une période qui se chiffre en années; enfin ils impliquent une pluralisation étiologique. Ces violences montrent que le marché des idées en matière de paradigme et de modélisation de ces phénomènes reste pauvre et nécessite un nouvel ensemble de propositions qui, peut-être, formeront à terme un consensus et l'ébauche d'un paradigme plus approprié. À cet égard, il nous semble utile de penser désormais non plus ces événements en termes de chaîne de commandement, mais bien plutôt de gouvernance (Foucault, 1978), où les processus qui mènent au massacre de centaines de milliers de victimes constituent le résultat de l'action conjointe d'un réseau d'acteurs et de dynamiques plutôt que d'un État unique agissant intentionnellement et rationnellement. Un tel cadre permettrait de réfléchir avec une perspective globale tenant compte d'un ensemble plus vaste de dimensions et d'acteurs. Les relations de pouvoir que les différents exécutants entretiennent les uns avec les autres, et également avec la population locale, leur position topographique par rapport aux atrocités commises, les allégeances de chacun, le degré d'autonomie dont ils disposent, constituent de nouvelles questions de recherche. Bref, les massacres de masse doivent désormais être pensés 
comme un champ de pratiques qui nécessite la création d'une approche davantage micro et axée sur le local, ainsi que le décloisonnement d'une pensée réaliste encore trop axée sur des outils analytiques dont l'utilité est limitée. Les notions de pluralisation d'acteurs, d'étiologies, de microrégulations, de relations entre les différents protagonistes de la violence doivent désormais être analysées plus systématiquement si l'on souhaite enrichir nos connaissances sur ces phénomènes et ainsi espérer contribuer à une désescalade de la violence.

\section{Références}

Andreopoulos, G. J. (1994). Genocide: Conceptual and Historical Dimensions. Philadelphia: University of Pennsylvania Press.

Arendt, H. (1963). Eichman à Jérusalem: rapport sur la banalité du mal. Paris: Gallimard.

Ball, H. (1999). Prosecuting War Crimes and Genocide: The Twentieth-Century Experience. Lawrence: University Press of Kansas.

Bassiouni, C. (1994). Final Report of the United Nations Commission of Experts Established Pursuant to Security Council Resolution 780 (1992) : Annex III : Special Units. New York: Nations Unies.

Bauman, Z. (1989). Modernity and the Holocaust. Ithaca, New York: Cornell University Press.

Bax, M. (2000a). Warlords, Priests and the Politic of Ethnic Cleansing: A Case Study from Rural Bosnie-Herzegovina. Etnic and Racial Studies, 23 (1), 16-36.

Bax, M. (2000b). Planned Policy or Primitive Balkanism? A Local Contribution to the Ethnography of the War in Bosnia-Herzegovina. Ethnos, 65 (3), 317-340.

Boudon, R. (1977). Effets pervers et ordre social. Paris: Presses universitaires de France.

Bougarel, X. (1996). Bosnie: anatomie d'un conflit. Paris: La Découverte.

Brauman, R. \& Sivan, E. (1999). Éloge de la désobéissance: à propos d'un «spécialiste» Adolf Eichmann. Paris: Le Pommier.

Browning, C. C. (1993). Ordinary Men: Reserve Police Battalion 101 and the Final Solution in Poland. New York: Harper Perennial.

Chalk, F. R. \& Jonassohn, K. (1990). The History and Sociology of Genocide: Analyses and Case Studies. New Haven: Yale University Press.

Charny, I. (1988). Genocide: A Critical Bibliographic Review. London: Mansell.

Denitch, B. (1996). Ethnic Nationalism: The Tragic Death of Yugoslavia. Minneapolis: University of Minnesota Press.

Fein, H. (1990). Genocide: A Sociological Perspective. London: Sage Publications.

Foucault, M. (1978). La gouvernementalité. In M. Foucault (Ed.), Dits et écrits, II: 1976-1988 (635-657). Paris : Quatro Gallimard. 
Goldhagen, D. J. (1997). Les bourreaux volontaires d'Hitler. Les Allemands ordinaires et l'Holocauste. Paris: Le Seuil.

Grmek, M., Gjidara, M. \& Simac, N. (1993). Le nettoyage ethnique: documents bistoriques sur une idéologie serbe. Paris: Point, Seuil.

Hatzfeld, J. (2003). Une saison de machettes, récits. Paris: Le Seuil.

Hayden, R. M. (2002). Imagined Communities and Real Victims: SelfDetermination and Ethnic Cleansing in Yugoslavia. In L. Hinton (Ed.), Genocide: An Anthropological Reader (231-253). Oxford: Blackwell.

Hilberg, R. (1985). La destruction des Juifs d'Europe. Paris: Gallimard.

Horowitz, I. L. (2002). Taking Lives: Genocide and State Power. New Brunswick (USA): Transaction Publishers.

Judah, T. (1997). The Serbs: History, Myth and the Destruction of Yugoslavia. New Haven: Yale University Press.

Kalyvas, S. N. (2003). The Ontology of Political Violence: Action and Identity in Civil Wars. Perspectives on Politics, 1 (3), 475-494.

Kalyvas, S. N. (2006) The Logic of Violence in Civil War. New York: Cambridge University Press.

Kressel, N. J. (2002). Mass Hate: The Global Rise of Genocide and Terror. Cambridge: Westview Press.

Kuhn, T. S. (1962). La structure des révolutions scientifiques. Paris: Champs Flammarion.

Kuper, L. (1981). Genocide: Its Political Use in the Twentieth Century. New Haven: Yale University Press.

Lemkin, R. (1944). Axis Rules in Occupied Europe. Washington, DC: Carnegie Endowment for International Peace.

Loyd, A. (1999). My War Gone By, I Miss It So. New York: Penguin Books.

Malcolm, N. (1994). Bosnia: A Short History. London: Papermac.

Mueller, J. (2000). The Banality of Ethnic War. International Security, 25 (2), 42-70.

Pisier, É., Châtelet, F., Duhamel, O., Bouretz, P., Colas, D. \& Guillarme, B. (2004). Histoire des idées politiques. Paris: Presses Universitaires de France.

Robertson, G. (1999). Crimes against Humanity: The Struggle for Global Justice. New York: The New Press.

Ron, J. (2000a). Territoriality and Plausible Deniability: Serbian Paramilitaries and the Bosnian War. In A. D. Brenner (Ed.), Death Squads in Global Perspective: Murder With Deniability (287-312). New York: St. Martin's Press.

Ron, J. (2000b). Boundaries and Violence: Repertoires of State Action along the Bosnia/Yugoslavia Divide. Theory and Society, 29, 609-649.

Schabas, W. A. (2000). Genocide in International Law: The Crime of Crimes. Cambridge: Cambridge University Press.

Schmitt, C. (1963). La notion de politique. Paris: Flammarion.

Sémelin, J. (2005). Purifier et détruire: usages politiques des massacres et génocides. Paris: Seuil. 
Silber, L. \& Little, A. (1995). The Death of Yugoslavia. New York: Penguin Books BBC Books.

Stroschein, S. (2005). Examining Ethnic Violence and Partition in BosniaHerzegovina. Ethnopolitics, 4 (1), 49-64.

Traverso, E. (2004). Une extermination moderne. Manière de voir, 76 (aoûtseptembre), 10-13.

Walliman, I. \& Dubkowski, M. (1987). Genocide and the Modern Age. Westport, CT: Greenwood Press.

Weber, M. (1921). Économie et société, vol. I: les catégories de la sociologie. Paris: Plon. 